\title{
Rider Trunk and Bicycle Pose Estimation With Fusion of Force/Inertial Sensors
}

\author{
Yizhai Zhang, Student Member, IEEE, Kuo Chen, and Jingang Yi*, Senior Member, IEEE
}

\begin{abstract}
Estimation of human pose in physical humanmachine interactions such as bicycling is challenging because of highly-dimensional human motion and lack of inexpensive, effective motion sensors. In this paper, we present a computational scheme to estimate both the rider trunk pose and the bicycle roll angle using only inertial and force sensors. The estimation scheme is built on a rider-bicycle dynamic model and the fusion of the wearable inertial sensors and the bicycle force sensors. We take advantages of the attractive properties of the robust force measurements and the motion-sensitive inertial measurements. The rider-bicycle dynamic model provides the underlying relationship between the force and the inertial measurements. The extended Kalman filter-based sensor fusion design fully incorporates the dynamic effects of the force measurements. The performance of the estimation scheme is demonstrated through extensive indoor and outdoor riding experiments.
\end{abstract}

Index Terms-Accelerometer and gyroscope, cycling, force sensor, motion and pose estimation, sensor fusion.

\section{INTRODUCTION}

$\mathbf{P}$ OSE and gait estimation not only benefits clinical analysis and diagnosis [1], but also provides tools to understand human sensorimotor mechanisms and their interactions with machines and environment. Human pose estimation in physical human-machine interactions such as bicycling is challenging because of the highly dimensional human movement and the dynamic interactions with machines. Further challenges arise in lack of effective, nonintrusive motion sensors in natural environment.

Existing motion capture systems, such as optical-, acoustic-, or magnetic-based tracking systems, are limited to indoor usage within a confined space and cannot be used for tracking human movement in natural environment. Wearable sensors have been extensively used for human pose and gait estimation [2]. For

Manuscript received January 14, 2013; revised April 6, 2013; accepted April 22, 2013. Date of publication April 26, 2013; date of current version August 16, 2013. This work was supported in part by the U.S. National Science Foundation under CAREER Award CMMI-0954966. This paper was presented in part at the 2013 American Control Conference, Washington, DC, USA, June 17-19, 2013. Asterisk indicates corresponding author.

Y. Zhang and K. Chen are with the Department of Mechanical and Aerospace Engineering, Rutgers University, Piscataway, NJ 08854 USA (e-mail: yzzhang@eden.rutgers.edu; kc625@eden.rutgers.edu).

* J. Yi is with the Department of Mechanical and Aerospace Engineering, Rutgers University, Piscataway, NJ 08854 USA (e-mail: jgyi@ rutgers.edu).

Color versions of one or more of the figures in this paper are available online at http://ieeexplore.ieee.org.

Digital Object Identifier 10.1109/TBME.2013.2260339 example, estimations of human upper-limb orientations were discussed in [3]-[6]. The work in [7]-[11] discusses the lowerlimb pose estimation. Some other works discuss general human segment pose measurement [12]-[15]. Most of the aforementioned work focuses on human walking, standing, or reaching activities, and there is few work that discusses the human motion and pose estimation in highly skilled human-machine interactions such as bicycling. The goal of this paper is to develop an estimation scheme for the rider trunk pose and the bicycle roll angle using inertial and force sensors.

Human bicycling motor skill is chosen as a pose estimation application for several reasons. Unlike commonly studied walking or stance where the human-environment interaction is only through the ground contact, the rider-bicycle interactions are through multiple contacts at the handlebar, the seat, and the pedals. The multicontact interactions bring complexity but also provide new features for pose estimation. Sitting on the unstable platform, riders have to actively react to the sensory feedback through body movement for balancing. Bicycles provide a unique platform for studying coupled human sensorimotor functions with machines. Recent clinical studies demonstrate promising results of treating Parkinson's disease patients through bicycle riding [16]-[18]. Bicycles can be further developed and used as a postural rehabilitation device.

Because of small size, low cost, and low power consumption, microelectromechanical-system-based inertial sensors such as accelerometers, gyroscopes, or inertial measurement units (IMUs) are widely used as wearable sensors for human motion and gait estimation. The orientation or the position of a body segment can be obtained by the integration of gyroscope or acceleration signals. However, the results of such strapdown IMU integration have the severe drifting problem due to the sensor measurement biases and noises. To overcome this problem, other complementary sensors are usually fused with inertial sensors to eliminate the drifting effect. For example, inclinations are estimated by accelerometer measurements and then integrated with gyroscope measurements in [6] and [13]. In [19], ultrasonic sensors are attached on human body to provide positioning information. In [12], [14], and [15], magnetic sensors are used as an attitude reference to constrain the drift growth and to provide the initial estimates in the filter design. However, the aforementioned sensor fusion schemes have various limitations. Accelerometer-based inclination can generate large measurement errors for dynamic activities, ultrasonic sensors potentially suffer from the line-of-sight restriction, and magnetic sensors are vulnerable to magnetic disturbances in environment. $\mathrm{Hu}-$ man anatomical constraints are also used to enhance the fusion 


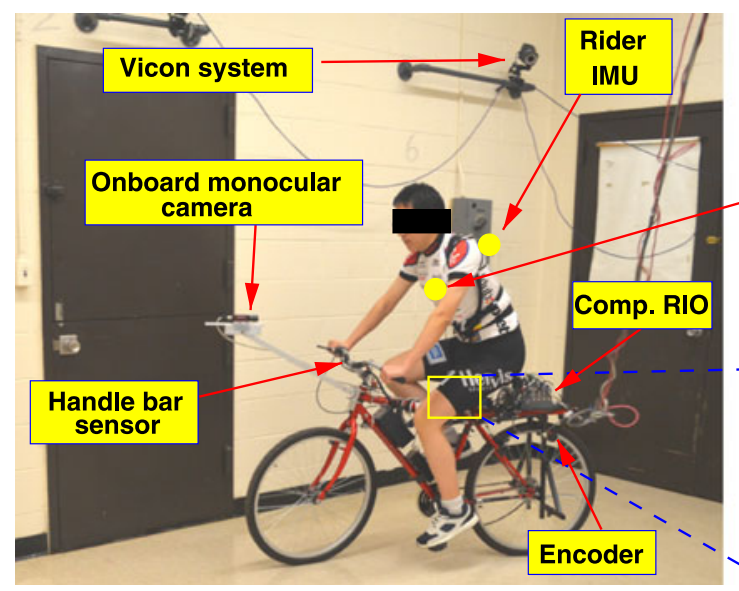

(a)

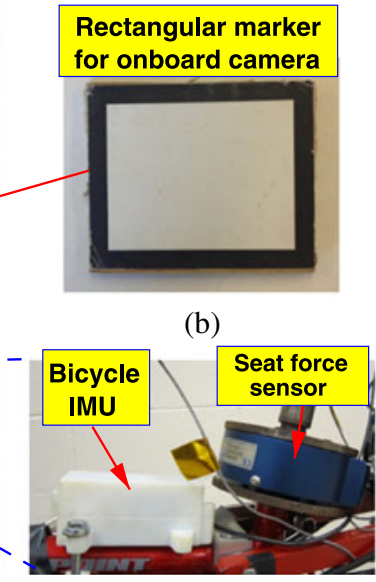

(c)

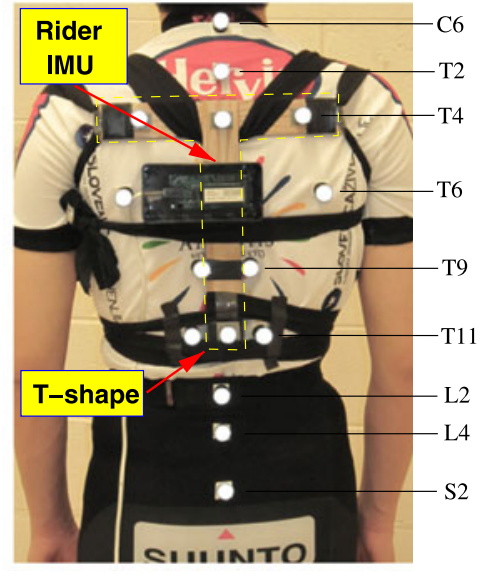

(d)

Fig. 1. (a) Instrumented bicycle. (b) Rectangular marker for outdoor use. (c) Bicycle IMU and seat force sensor. (d) Locations of the IMU and optical markers mounted on rider trunk.

accuracy [5]. In [11], multiple accelerometers are used to estimate the gait without directly integrating IMU measurements. For walking gait estimation, a "resetting" technique is used in [8] and [20] to initialize the integration at the beginning of each stride.

In this paper, we fuse the force sensors with the IMUs to overcome the drifting issue in integration of the inertial measurements. Unlike magnetic and ultrasonic sensors, the force sensors are reliable and robust to environmental disturbances and do not have the line-of-sight restriction. The fusions of force and inertial sensors have been used to measure the interaction kinetics between human and environment [21]-[24]. However, the work in [21]-[24] uses the force and inertial sensors to obtain kinetic measurements and none of them uses the force measurements to enhance the pose estimation. Unlike in walking or stance, pose estimation in bicycling has different features. The rider sits on a moving platform and the measurements from wearable IMUs contain motion information of both the rider and the bicycle. Multiple IMUs are needed and analyses have to be conducted to decouple the IMU measurements. A trunkbicycle dynamic model is used to reveal the underlying dynamic relationship between the inertial and force measurements. We present extensive experiments to demonstrate the drift-free and robust performance of the pose estimation design. This paper is a significant extension of the previously presented conference publication [25].

The main contributions of this paper are twofold. First, the pose estimation scheme relies only on the wearable sensors and the onboard sensors, and therefore, it aims for human pose and gait studies in daily surroundings rather than in restrictive indoor environment. The use of the inertial/force sensors fusion is novel and provides reliable and robust estimates of human pose under dynamic motions. Second, the new dynamic model captures the rider-bicycle motion characteristics and bridges the human motion kinematics with the driving force measurements. The modeling framework provides a new approach to potentially study other types of human-machine interactions.

\section{METHODS}

\section{A. Instrumented Bicycle and Riding Experiments}

Fig. 1(a) shows the instrumented bicycle. The bicycle is modified from a commercial mountain bike and equipped with various sensors. The bicycle is designed for both indoor and outdoor experiments. A force/torque sensor (from JR3 Inc.) is installed along the seat supporting rod to measure the three-axis hip-seat forces and torques (see Fig. 1(c)). Three load cells are installed inside the customly built bicycle seat to measure the sitting force distribution (see Fig. 3). An optical encoder is used to measure the bicycle speed. A set of stain gauges are installed on the bicycle handlebar to measure the handlebar forces. A real-time embedded system (CompactRIO 9074 from National Instruments Inc.) samples and stores all sensor measurements at the frequency of $50 \mathrm{~Hz}$, a maximum sampling frequency that can be achieved by the given hardware capability.

Two IMU units (model 605 from Motion Sense Inc. ${ }^{1}$ ) are used: one IMU is mounted to the bicycle frame (see Fig. 1(c)) and the other one is rigidly attached to a T-shape fixture at level T6 on the back of the rider trunk. The T-shape fixture spans levels T4 to T11 (see Fig. 1(d)). Each IMU consists of a triaxial gyroscope and a triaxial accelerometer. Similar to [26]-[28], we assume the rigid-body movement of the trunk and we will discuss this assumption in Section IV. The direction of the spinal segment from levels T4 to T11 is used to represent the human trunk orientation.

For indoor experiments, a vision-based motion capture system (from Vicon Inc.) is used to provide the ground truth for the trunk and bicycle poses. The Vicon system includes eight Botina cameras, MX Giganet module, and a workstation computer with Nexus 1.6 motion capture software. For outdoor experiments, the ground truth of the bicycle attitude angles is obtained by a high-accuracy IMU (model 800 from Motion Sense Inc.). The trunk pose with respect to the bicycle is obtained by an onboard

\footnotetext{
${ }^{1}$ http://www.motionsense.com/
} 


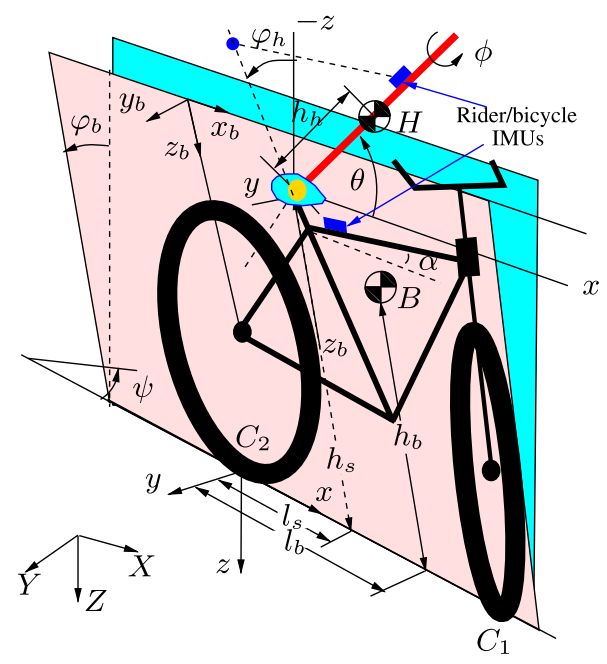

Fig. 2. Schematic of the rider-bicycle system.

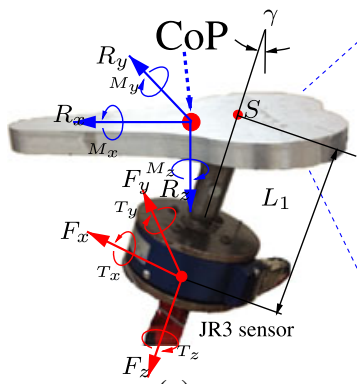

(a)

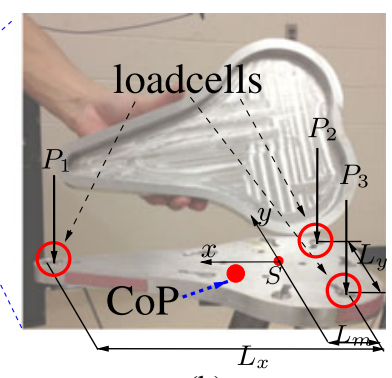

(b)
Fig. 3. (a) Schematic of bicycle seat forces/torques and the transformation. (b) CoP calculations through three load cell-measured forces.

high-resolution monocular camera with a rectangular-shape feature marker (see Fig. 1(b)). The camera (Manta G-145 from Allied Vision Technologies, $1392 \times 1040$ pixels, 16 frames/s) is mounted on an extended rod that is rigidly connected to the bicycle frame. The details of the camera-based pose calculation are given in [29]. The motion capturing systems are synchronized with the onboard sensors through the wireless network connections.

In experiments, the subjects are asked to ride the bicycle at their own riding styles. For indoor experiments, due to spatial constraints, the subjects are asked to ride the bicycle for a circular trajectory (with radius around $2.5 \mathrm{~m}$ ). For outdoor experiments, the subjects arbitrarily ride the bicycle in an area of a size of $50 \mathrm{~m} \times 40 \mathrm{~m}$. The ground is paved with bricks in outdoor experiment.

In the following, we first describe the models for the IMU and the rider-bicycle interactions. Then, we discuss the riderbicycle interaction forces. Finally, an extended Kalman filter (EKF) is designed to fuse the sensor measurements and predict the trunk pose and the bicycle roll angle.

\section{B. IMU Model}

Fig. 2 illustrates a schematic of the kinematic rider-bicycle interactions. The rider's trunk is modeled as an inverted pendulum in the 3-D space. A ground-fixed inertial frame $\mathcal{I}(X, Y, Z)$ is defined with the $Z$-axis pointing downward. A moving frame $\mathcal{R}(x, y, z)$ is defined with the $x$-axis along wheel-ground contact points $C_{1}$ and $C_{2}$, the $z$-axis along the $Z$-axis, and the origin at $C_{2}$. The bicycle roll and yaw angles are denoted as $\varphi_{b}$ and $\psi$, respectively. A bicycle-fixed frame $\mathcal{B}\left(x_{b}, y_{b}, z_{b}\right)$ is obtained by rotating frame $\mathcal{R}$ with $\varphi_{b}$ about the $x$-axis (see Fig. 2).

The IMU on the bicycle frame is tilted by angle $\alpha$ with respect to the $x_{b}$-axis. Let $\mathcal{I}_{h}$ and $\mathcal{I}_{b}$ denote the rider and bicycle IMU frames, respectively. The orientation of the trunk is defined by three Euler angles with the $X-Y-X$ ordered rotation from $\mathcal{R}$ to $\mathcal{I}_{h}$ : roll angle $\varphi_{h}$ around the $x$-axis, angle $\theta$ around the $y$-axis, and finally, self-spinning angle $\phi$ around the $x$-axis. The generalized coordinates for the trunk and the bicycle are denoted as $\boldsymbol{q}_{h}=\left[\varphi_{h} \theta \phi\right]^{T}$ and $\boldsymbol{q}_{b}=\left[\varphi_{b} \psi\right]^{T}$, respectively. We also define $\boldsymbol{q}=\left[\begin{array}{ll}\boldsymbol{q}_{h}^{T} & \boldsymbol{q}_{b}^{T}\end{array}\right]^{T}$.

1) Gyroscope Model: The bicycle IMU gyroscope measurements $\boldsymbol{\omega}_{b}=\left[\begin{array}{lll}\omega_{b x} & \omega_{b y} & \omega_{b z}\end{array}\right]^{T}$ are calculated as

$$
\boldsymbol{\omega}_{b}=\boldsymbol{R}_{y}^{T}(\alpha) \boldsymbol{R}_{x}^{T}\left(\varphi_{b}\right)\left[\begin{array}{c}
0 \\
0 \\
\dot{\psi}
\end{array}\right]+\boldsymbol{R}_{y}^{T}(\alpha)\left[\begin{array}{c}
\dot{\varphi}_{b} \\
0 \\
0
\end{array}\right],
$$

where $\boldsymbol{R}_{i}(\beta)$ represents the 3-D rotational matrix around the $i$-axis with angle $\beta, i=x, y, z .^{2}$ To obtain the rider IMU gyroscope model, we consider the transformation from $\mathcal{R}$ to $\mathcal{I}_{h}$ and the rider IMU gyroscope measurements $\boldsymbol{\omega}_{h}=$ $\left[\begin{array}{lll}\omega_{h x} & \omega_{h y} & \omega_{h z}\end{array}\right]^{T}$ are then calculated as

$$
\begin{aligned}
\boldsymbol{\omega}_{h}= & \boldsymbol{R}_{x}^{T}(\phi) \boldsymbol{R}_{y}^{T}(\theta)\left[\begin{array}{c}
\dot{\varphi}_{h} \\
0 \\
0
\end{array}\right]+\boldsymbol{R}_{x}^{T}(\phi)\left[\begin{array}{c}
0 \\
\dot{\theta} \\
0
\end{array}\right]+\left[\begin{array}{c}
\dot{\phi} \\
0 \\
0
\end{array}\right] \\
& +\boldsymbol{R}_{x}^{T}(\phi) \boldsymbol{R}_{y}^{T}(\theta) \boldsymbol{R}_{x}^{T}\left(\varphi_{h}\right)\left[\begin{array}{c}
0 \\
0 \\
\dot{\psi}
\end{array}\right]
\end{aligned}
$$

Combining (1) and (2) and solving for $\dot{\boldsymbol{q}}_{b}$ and $\dot{\boldsymbol{q}}_{h}$, we obtain

$$
\begin{aligned}
\dot{\boldsymbol{q}}_{b}= & {\left[\begin{array}{c}
\dot{\varphi}_{b} \\
\dot{\psi}
\end{array}\right]=\boldsymbol{e}\left(\boldsymbol{q}_{b} ; \boldsymbol{\omega}_{b}\right)=\left[\begin{array}{ccc}
\mathrm{c}_{\alpha} & 0 & \mathrm{~s}_{\alpha} \\
-\frac{\mathrm{s}_{\alpha}}{\mathrm{c}_{\varphi_{b}}} & 0 & \frac{\mathrm{c}_{\alpha}}{\mathrm{c}_{\varphi_{b}}}
\end{array}\right] \boldsymbol{\omega}_{b} } \\
\dot{\boldsymbol{q}}_{h}= & {\left[\begin{array}{c}
\dot{\varphi} h \\
\dot{\theta} \\
\dot{\phi}
\end{array}\right]=\boldsymbol{f}\left(\boldsymbol{q} ; \boldsymbol{\omega}_{h}, \boldsymbol{\omega}_{b}\right)=\left[\begin{array}{ccc}
0 & \frac{\mathrm{s}_{\phi}}{\mathrm{s}_{\theta}} & \frac{\mathrm{c}_{\phi}}{\mathrm{s}_{\theta}} \\
0 & \mathrm{c}_{\phi} & -\mathrm{s}_{\phi} \\
1 & -\frac{\mathrm{c}_{\theta}}{\mathrm{s}_{\theta}} \mathrm{s}_{\phi} & -\frac{\mathrm{c}_{\theta}}{\mathrm{s}_{\theta}} \mathrm{c}_{\phi}
\end{array}\right] \boldsymbol{\omega}_{h} } \\
+ & {\left[\begin{array}{ccc}
\frac{\mathrm{s}_{\alpha} \mathrm{c}_{\theta} \mathrm{c}_{\varphi_{h}}}{\mathrm{c}_{\varphi_{b}} \mathrm{~s}_{\theta}} & 0 & -\frac{\mathrm{c}_{\alpha} \mathrm{c}_{\theta} \mathrm{c}_{\varphi_{h}}}{\mathrm{c}_{\varphi_{b}} \mathrm{~s}_{\theta}} \\
\frac{\mathrm{s}_{\alpha} \mathrm{s}_{\varphi_{h}}}{\mathrm{c}_{\varphi_{b}}} & 0 & -\frac{\mathrm{c}_{\alpha} \mathrm{s}_{\varphi_{h}}}{\mathrm{c}_{\varphi_{b}}} \\
-\frac{\mathrm{s}_{\alpha} \mathrm{c}_{\varphi_{h}}}{\mathrm{c}_{\varphi_{b}} \mathrm{~s}_{\theta}} & 0 & \frac{\mathrm{c}_{\alpha} \mathrm{c}_{\varphi_{h}}}{\mathrm{c}_{\varphi_{b}} \mathrm{~s}_{\theta}}
\end{array}\right] \boldsymbol{\omega}_{b}, }
\end{aligned}
$$

\footnotetext{
${ }^{2}$ The details of the definition and formulation of the standard rotational matrices can be found in [30] (e.g., p. 31) and we omit here.
} 
where notations $\mathrm{c}_{\varphi_{h}}=\cos \varphi_{h}, \mathrm{~S}_{\varphi_{h}}=\sin \varphi_{h}$ are used for angle $\varphi_{h}$ and any other angles throughout this paper.

2) Accelerometer Model: The position of the bicycle IMU in $\mathcal{B}$ is denoted as $\boldsymbol{r}_{b I}=\left[\begin{array}{lll}p_{x} & 0 & -p_{z}\end{array}\right]^{T}$, where $p_{x}$ and $p_{z}$ are the horizontal and vertical distances from the IMU to $C_{2}$, respectively. Considering the nonholonomic constraint at $C_{2}$, the acceleration of $C_{2}$ in $\mathcal{R}$ is ${ }^{\mathcal{R}} \dot{\boldsymbol{v}}_{C_{2}}=\left[\begin{array}{lll}\dot{v}_{b} & 0 & g\end{array}\right]^{T}$, where $g$ is the gravitational constant and $v_{b}$ is the bicycle velocity. The angular velocity of the bicycle in $\mathcal{R}$ is ${ }^{\mathcal{R}} \boldsymbol{\omega}_{b}=\left[\begin{array}{lll}\dot{\varphi}_{b} & 0 & \dot{\psi}\end{array}\right]^{T}$. The bicycle IMU accelerometer measurements $\boldsymbol{a}_{b}=\left[\begin{array}{lll}a_{b x} & a_{b y} & a_{b z}\end{array}\right]^{T}$ in $\mathcal{I}_{b}$ are then calculated as

$\boldsymbol{a}_{b}={ }_{\mathcal{I}_{b}}^{\mathcal{R}} \boldsymbol{R}^{T}\left[{ }^{\mathcal{R}} \dot{\boldsymbol{v}}_{C_{2}}+{ }^{\mathcal{R}} \boldsymbol{\omega}_{b} \times{ }^{\mathcal{R}} \boldsymbol{\omega}_{b} \times{ }_{\mathcal{B}}^{\mathcal{R}} \boldsymbol{R} \boldsymbol{r}_{b I}+{ }^{\mathcal{R}} \dot{\boldsymbol{\omega}}_{b} \times{ }_{\mathcal{B}}^{\mathcal{R}} \boldsymbol{R} \boldsymbol{r}_{b I}\right]$,

where $_{\mathcal{B}}^{\mathcal{R}} \boldsymbol{R}=\boldsymbol{R}_{x}\left(\varphi_{b}\right)$ and ${ }_{\mathcal{I}_{b}}^{\mathcal{R}} \boldsymbol{R}^{T}=\boldsymbol{R}_{y}^{T}(\alpha) \boldsymbol{R}_{x}^{T}\left(\varphi_{b}\right)$ are the rotational matrices from $\mathcal{R}$ to $\mathcal{B}$ and $\mathcal{I}_{b}$, respectively. The calculation of $\boldsymbol{a}_{b}$ is given in (6) at the bottom of the page. Notice that the coefficients of $\ddot{\psi}$ in $a_{b x}$ and $a_{b z}$ are near zero when $\varphi_{b}$ is around zero. We take an approximation that during typically bicycle riding, the average value of $\ddot{\varphi}_{b}$ is around zero, i.e., $\ddot{\varphi}_{b} \approx 0$. From $a_{b y}$ in (6), we then obtain

$$
\ddot{\psi}=\frac{a_{b y}}{p_{x} \mathrm{c}_{\varphi_{b}}}-\frac{\mathrm{s}_{\varphi_{b}} p_{z}}{p_{x}} \dot{\psi}^{2}-\frac{\mathrm{s}_{\varphi_{b}}}{\mathrm{c}_{\varphi_{b}}} \dot{\psi} \dot{\varphi}_{b}-\frac{\mathrm{s}_{\varphi_{b}} g}{\mathrm{c}_{\varphi_{b}} p_{x}} .
$$

Let $\boldsymbol{r}_{s}=\left[\begin{array}{lll}l_{s} & 0 & -h_{s}\end{array}\right]^{T}$ denote the seat position in $\mathcal{B}$, where $l_{s}$ and $h_{s}$ are the horizontal and vertical distances from the seat to $C_{2}$, respectively (see Fig. 2). To make calculation tractable, the angular velocity of rider's trunk in $\mathcal{R}$ is approximated as ${ }^{\mathcal{R}} \boldsymbol{\omega}_{h}=\left[\begin{array}{lll}\dot{\varphi}_{h} & c_{\varphi_{h}} \dot{\theta} \dot{\psi}+\mathrm{s}_{\varphi_{h}} \dot{\theta}\end{array}\right]^{T}$. Let $\boldsymbol{r}_{h I}=\left[\begin{array}{lll}h & 0 & 0\end{array}\right]^{T}$ denote the position of the rider IMU in $\mathcal{I}_{h}$ with respect to the seat, where $h$ is the distance from the rider IMU to the seat. Similar to (5), we obtain the seat acceleration ${ }^{\mathcal{R}} \boldsymbol{a}_{s}=\left[\begin{array}{lll}a_{s x} & a_{s y} & a_{s z}\end{array}\right]^{T}$ in $\mathcal{R}$ and the rider IMU accelerometer measurements $\boldsymbol{a}_{h}=\left[\begin{array}{lll}a_{h x} & a_{h y} & a_{h z}\end{array}\right]^{T}$ are then calculated using ${ }^{\mathcal{R}} \boldsymbol{a}_{s}$. Similar to (7), we obtain the attitude acceleration formulation in (8) at the bottom of the page by using acceleration $\boldsymbol{a}_{h}$.

With the results in (7) and (8), the IMU accelerometer measurements $\boldsymbol{a}_{h}$ and $\boldsymbol{a}_{b}$ are used to provide the attitude accelerations $\ddot{\varphi}_{h}, \ddot{\theta}$, and $\ddot{\psi}$ in the EKF design in Section II-E.

\section{Rider-Bicycle Dynamic Model}

Let $\boldsymbol{\rho}_{B}=\left[\begin{array}{lll}l_{b} & 0 & -h_{b}\end{array}\right]^{T}$ denote the position of bicycle mass center $B$ in $\mathcal{B}$, where $l_{b}$ and $h_{b}$ are the horizontal and the vertical distances from $C_{2}$ to $B$, respectively. The trunk mass is denoted as $m_{h}$ at $H$ (see Fig. 2). The distance between $H$ and the seat is denoted as $h_{h}$. Using Lagrange's equations, we obtain the human driving torque

$$
\boldsymbol{\tau}_{h}=\left[\begin{array}{c}
\tau_{\varphi_{h}} \\
\tau_{\theta}
\end{array}\right]=\boldsymbol{M} \ddot{\boldsymbol{q}}_{h}+\boldsymbol{C}\left(\boldsymbol{q}, \dot{\boldsymbol{q}}, \ddot{\boldsymbol{q}}_{b}\right)+\boldsymbol{G},
$$

where $\tau_{\varphi_{h}}$ and $\tau_{\theta}$ are the driving torques along the $x$-axis $\left(\varphi_{h}\right)$ and the $y^{\prime}$-axis $(\theta)$ directions in $\mathcal{R}$, respectively. The $y^{\prime}$-axis is obtained from the $y$-axis by rotating $\varphi_{h}$ about the $x$-axis. A brief derivation of (9) and matrices $\boldsymbol{M}, \boldsymbol{C}\left(\boldsymbol{q}, \dot{\boldsymbol{q}}, \ddot{\boldsymbol{q}}_{b}\right)$ and $\boldsymbol{G}$ are given in Appendix A.

\section{Force/Torque Sensor Model}

We designed and fabricated a special seat as shown in Fig. 3. Three load cells are used to calculate the location of the center of pressure (CoP) of the hip-seat interaction force. In this section, we discuss how to use the force sensor measurements to obtain driving torque $\boldsymbol{\tau}_{h}$ in (9).

A seat frame $\mathcal{S}_{t}$ is defined as the same orientation of $\mathcal{B}$ with the origin $S$ at the intersection of the seat rod and the seat surface [see Fig. 3(a)]. Let $\boldsymbol{R}_{h}=\left[\begin{array}{lll}R_{x} & R_{y} & R_{z}\end{array}\right]^{T}$ and $\boldsymbol{M}_{h}=\left[\begin{array}{lll}M_{x} & M_{y} & M_{z}\end{array}\right]^{T}$ denote the hip-seat forces (acting at the $\mathrm{CoP})$ and torques in $\mathcal{S}_{t}$, respectively. Let $\boldsymbol{F}_{s}=\left[\begin{array}{lll}F_{x} & F_{y} & F_{z}\end{array}\right]^{T}$ and $\boldsymbol{T}_{s}=\left[T_{x} T_{y} T_{z}\right]^{T}$ denote the forces and torques measured by the JR 3 sensor in sensor frame $\mathcal{S}_{r}$, respectively. Considering the transformation between frames $\mathcal{S}_{t}$ and $\mathcal{S}_{r}$, we obtain

$$
\left[\begin{array}{c}
\boldsymbol{R}_{h} \\
\boldsymbol{M}_{h}
\end{array}\right]=\left[\begin{array}{cc}
\mathcal{S}_{\mathcal{S}_{r}} \boldsymbol{R} & \mathbf{0} \\
\boldsymbol{S}\left(\mathcal{S}^{\mathcal{S}_{t}} \boldsymbol{r}_{c}\right)_{\mathcal{S}_{r}}^{\mathcal{S}_{t}} \boldsymbol{R} & \mathcal{S}_{\mathcal{S}_{r}} \boldsymbol{R}
\end{array}\right]\left[\begin{array}{c}
\boldsymbol{F}_{s} \\
\boldsymbol{T}_{s}
\end{array}\right],
$$

where ${ }_{\mathcal{S}_{r}} \boldsymbol{S}_{t}=\boldsymbol{R}_{y}(\gamma)$ is the rotational matrix from frames $\mathcal{S}_{t}$ to $\mathcal{S}_{r}$ and $\gamma$ is the tilting angle of the supporting rod. The skew-symmetric matrix $\boldsymbol{S}\left({ }^{\mathcal{S}_{t}} \boldsymbol{r}_{c}\right)$ is defined by ${ }^{\mathcal{S}_{t}} \boldsymbol{r}_{c}=\left[-x_{C}+\right.$ $\left.\begin{array}{lllll}L_{1} & \mathrm{~s}_{\gamma} & -y_{C} & L_{1} & \mathrm{c}_{\gamma}\end{array}\right]^{T}$ (the position vector of the force sensor with respect to the CoP in $\mathcal{S}_{t}$ ), $L_{1}$ is the distance between $S$ and the JR3 sensor, and $\left(x_{C}, y_{C}\right)$ is the coordinate of the CoP in $\mathcal{S}_{t}$. Letting $P_{i}, i=1,2,3$, denote the measurements of the three load cells, $\left(x_{C}, y_{C}\right)$ is then calculated by

$$
x_{C}=\frac{P_{1}}{P_{1}+P_{2}+P_{3}} L_{x}-L_{m}, y_{C}=\frac{P_{2}-P_{3}}{2\left(P_{1}+P_{2}+P_{3}\right)} L_{y},
$$

where $L_{x}$ and $L_{y}$ are the $x$ - and $y$-axis directional distances between the front and rear load cells, respectively, and $L_{m}$ is the $x$-axis distance between $S$ and rear two load cells.

$$
\boldsymbol{a}_{b}=\left[\begin{array}{c}
\mathrm{c}_{\alpha} \dot{v}_{b}+\left(\mathrm{c}_{\alpha} \mathrm{c}_{\varphi_{b}} p_{z}-\mathrm{s}_{\alpha} \mathrm{c}_{\varphi_{b}} p_{x}\right) \dot{\psi} \dot{\varphi}_{b}+\left(\mathrm{s}_{\alpha} \mathrm{s}_{\varphi_{b}}^{2} p_{z}-\mathrm{c}_{\alpha} p_{x}\right) \dot{\psi}^{2}+\mathrm{s}_{\alpha} p_{z} \dot{\varphi}_{b}^{2}+\left(\mathrm{c}_{\alpha} \mathrm{s}_{\varphi_{b}} p_{z}+\mathrm{s}_{\alpha} \mathrm{s}_{\varphi_{b}} p_{x}\right) \ddot{\psi}-\mathrm{s}_{\alpha} \mathrm{c}_{\varphi_{b}} g \\
\mathrm{c}_{\varphi_{b}} \mathrm{~s}_{\varphi_{b}} p_{z} \dot{\psi}^{2}+\mathrm{c}_{\varphi_{b}} p_{x} \ddot{\psi}-p_{z} \ddot{\varphi}_{b}+\mathrm{s}_{\varphi_{b}} p_{x} \dot{\psi} \dot{\varphi}_{b}+\mathrm{s}_{\varphi_{b}} g \\
\mathrm{~s}_{\alpha} \dot{v}_{b}+\left(\mathrm{s}_{\alpha} c_{\varphi_{b}} p_{z}+\mathrm{c}_{\alpha} c_{\varphi_{b}} p_{x}\right) \dot{\psi} \dot{\varphi}_{b}-\left(\mathrm{c}_{\alpha} \mathrm{s}_{\varphi_{b}}^{2} p_{z}+\mathrm{s}_{\alpha} p_{x}\right) \dot{\psi}^{2}-\mathrm{s}_{\alpha} p_{z} \dot{\varphi}_{b}^{2}+\left(\mathrm{s}_{\alpha} \mathrm{s}_{\varphi_{b}} p_{z}-\mathrm{c}_{\alpha} \mathrm{s}_{\varphi_{b}} p_{x}\right) \ddot{\psi}+\mathrm{c}_{\alpha} \mathrm{c}_{\varphi_{b}} g
\end{array}\right]
$$

$$
\left[\begin{array}{c}
\ddot{\varphi} h \\
\ddot{\theta}
\end{array}\right]=\left[\begin{array}{c}
\frac{1}{\mathrm{~s}_{\theta} h} a_{h y}+\mathrm{s}_{\varphi_{h}} \mathrm{c}_{\varphi_{h}} \dot{\psi}^{2}+\mathrm{c}_{\varphi_{h}} \dot{\psi} \dot{\theta}-\mathrm{s}_{\varphi_{h}} \frac{\mathrm{c}_{\theta}}{\mathrm{s}_{\theta}} \dot{\varphi} h \dot{\psi}-\frac{\mathrm{c}_{\theta}}{\mathrm{s}_{\theta}} \dot{\varphi}_{h} \dot{\theta}-\mathrm{c}_{\varphi_{h}} \frac{\mathrm{c}_{\theta}}{\mathrm{s}_{\theta}} \ddot{\psi}-\frac{\mathrm{c}_{\varphi_{h}}}{\mathrm{~s}_{\theta} h} a_{s y}-\frac{\mathrm{s}_{\varphi_{h}}}{\mathrm{~s}_{\theta} h} a_{s z} \\
-\frac{a_{h z}}{h}-\mathrm{c}_{\varphi_{h}} \dot{\varphi}_{h} \dot{\psi}+2 \mathrm{c}_{\varphi_{h}} \mathrm{c}_{\theta}^{2} \dot{\varphi}_{h} \dot{\psi}-\mathrm{s}_{\theta} \mathrm{c}_{\theta} \mathrm{c}_{\varphi_{h}}^{2} \dot{\psi}^{2}+\mathrm{s}_{\theta} \mathrm{c}_{\theta} \dot{\varphi}_{h}^{2}-\mathrm{s}_{\varphi_{h}} \ddot{\psi}+\frac{\mathrm{s}_{\theta} a_{s x}}{h}-\frac{\mathrm{c}_{\theta} \mathrm{s}_{\varphi_{h}} a_{s y}}{h}+\frac{\mathrm{c}_{\varphi_{h}} a_{s z} \mathrm{c}_{\theta}}{h}
\end{array}\right]
$$




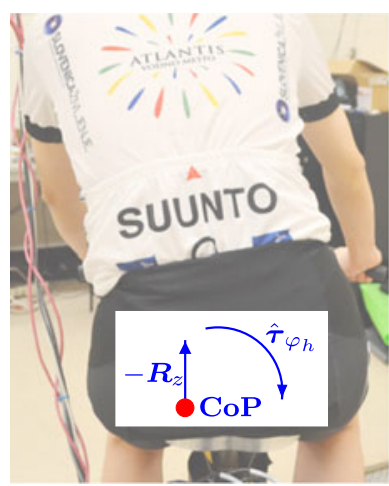

(a)

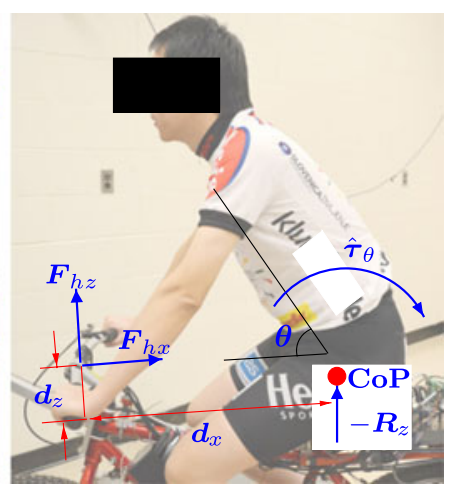

(b)
Fig. 4. Rider trunk driving forces and torques. (a) $\tau_{\varphi_{h}}$ calculation. (b) $\tau_{\theta}$ calculation.

To capture the sitting position variations among different riders, we define the CoP location $\boldsymbol{r}_{V}=\left[x_{V}, y_{V}\right]^{T}$ in $\mathcal{S}_{t}$ when the trunk is upright. Vector $\boldsymbol{r}_{V}$ is obtained for each subject in experiments. As shown in Fig. 4(a), along the $\varphi_{h}$-direction, the torque applied on the trunk is the sum of the measured $M_{x}$ and the torque generated by the reacting force $R_{z}$. Thus, we obtain the estimate of $\tau_{\varphi_{h}}$ as

$$
\hat{\tau}_{\varphi_{h}}=-M_{x}-R_{z}\left(y_{C}-y_{V}\right) .
$$

Along the $\theta$-direction, the handlebar forces also contribute to torque $\tau_{\theta}$ that drives the trunk motion (see Fig. 4(b)). Neglecting the arms dynamics, the estimated torque $\hat{\tau}_{\theta}$ is approximated as

$$
\hat{\tau}_{\theta}=\frac{-M_{y}+F_{h x} d_{z}+F_{h z} d_{x}-R_{z}\left(x_{C}-x_{V}\right)}{c_{\varphi_{b}-\varphi_{h}}},
$$

where $F_{h x}$ and $F_{h z}$ are the resultant handlebar reaction forces along the $x_{b}$-axis and the $z_{b}$-axis directions, respectively, and $d_{x}$ and $d_{z}$ are the horizontal and vertical distances from the handlebar to the seat, respectively. Finally, similar to the results in [28], bicycle roll angle $\varphi_{b}$ is estimated as

$$
\hat{\varphi}_{b}=\tan ^{-1}\left(\frac{R_{y}}{R_{z}}\right) .
$$

\section{E. EKF Design}

Fig. 5 illustrates the EKF design structure. The EKF system equations are built on the IMU gyroscope model with a bias model that will be discussed later in this section. The riderbicycle dynamic model is used as output equations to bridge the EKF state variables with the force sensor model. The IMU accelerometer model provides the attitude acceleration calculations to the rider-bicycle dynamic model.

A first-order random walk is considered for modeling the measurement bias only for the rider IMU gyroscope. If we consider the bias model for the both IMU gyroscopes, the EKF system becomes unobservable and the EKF convergence cannot be guaranteed. Let $\boldsymbol{n}_{h}$ and $\boldsymbol{n}_{b}$ denote the measurement noises for the rider and bicycle IMU gyroscopes, respectively. Then,

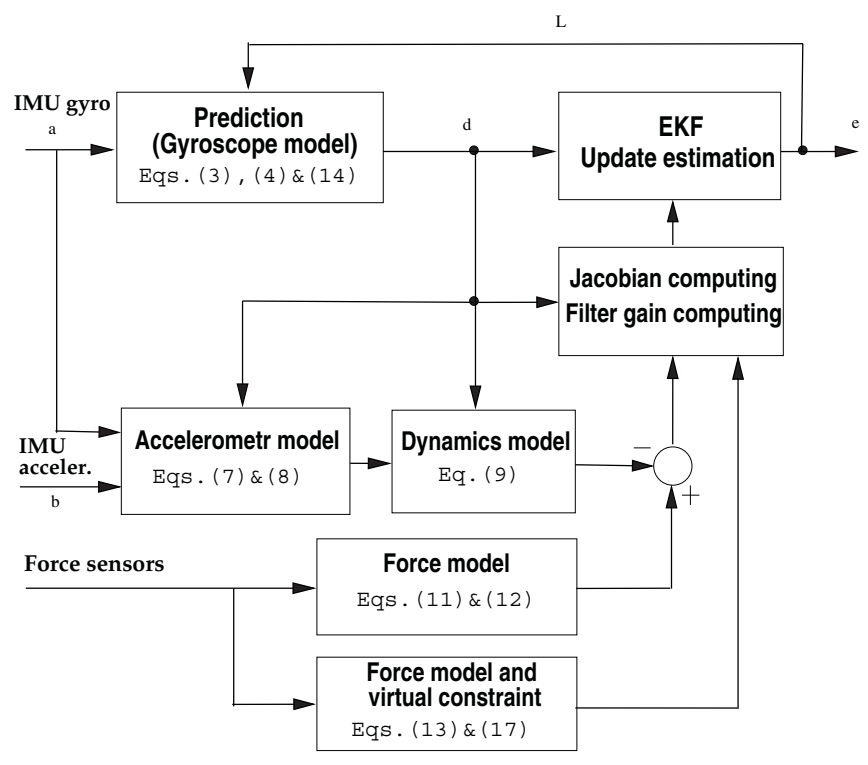

Fig. 5. Structural and information flow diagram of the EKF design.

we have the noise model [31]

$$
\boldsymbol{n}_{h}=\boldsymbol{c}_{h}+\boldsymbol{b}+\boldsymbol{\nu}_{h}, \boldsymbol{n}_{b}=\boldsymbol{c}_{b}+\boldsymbol{\nu}_{b},
$$

where $c_{h}$ and $c_{b}$ are the constant offset biases and obtained in experiments, and $\boldsymbol{\nu}_{h}$ and $\boldsymbol{\nu}_{b}$ are assumed to be the zero-mean white noise vectors. Vector $\boldsymbol{b}=\left[\begin{array}{lll}b_{x} & b_{y} & b_{z}\end{array}\right]^{T}$ is the random walk bias with the model

$$
\dot{b}_{i}=-\frac{1}{\tau_{i}} b_{i}+\sqrt{\frac{2 f_{s} \sigma_{i}^{2}}{\tau_{i}}} w_{i}, \quad i=x, y, z,
$$

where $\tau_{i}$ are the time constants, $f_{s}$ is the sampling frequency, $\sigma_{i}^{2}=E\left[b_{i}^{2}\right]$, and $w_{i} \sim \mathcal{N}(0,1)$ are zero-mean white noises with unit variances.

We define the discrete-time EKF state variables $\boldsymbol{X}(k)=$ $\left[\varphi_{h}(k) \theta(k) \phi(k) \varphi_{b}(k) b_{x}(k) b_{y}(k) b_{z}(k)\right]^{T}$ and

$$
\boldsymbol{f}_{x}(\boldsymbol{X}(k), \boldsymbol{u}(k))=\left[\begin{array}{c}
\boldsymbol{f}(\boldsymbol{q}(k) ; \boldsymbol{u}(k)) \\
e_{1}\left(\boldsymbol{X}(k) ; \boldsymbol{\omega}_{b}(k)\right) \\
-\frac{1}{\tau_{x}} b_{x}(k) \\
-\frac{1}{\tau_{y}} b_{y}(k) \\
-\frac{1}{\tau_{z}} b_{z}(k)
\end{array}\right],
$$

where $\boldsymbol{u}(k)=\left[\boldsymbol{\omega}_{h}^{T}(k) \boldsymbol{\omega}_{b}^{T}(k)\right]^{T}$ are the IMU gyroscope measurements at the $k$ th step, $\boldsymbol{f}(\boldsymbol{q}(k) ; \boldsymbol{u}(k))=\boldsymbol{f}\left(\boldsymbol{q}(k) ; \boldsymbol{\omega}_{h}(k)-\right.$ $\left.\boldsymbol{b}(k)-\boldsymbol{c}_{h}, \boldsymbol{\omega}_{b}(k)-\boldsymbol{c}_{b}\right)$ is given in (4), and $e_{1}\left(\boldsymbol{X}(k) ; \boldsymbol{\omega}_{b}(k)\right)$ is the first element of $\boldsymbol{e}\left(\boldsymbol{q}_{b} ; \boldsymbol{\omega}_{b}\right)$ in (3). We obtain the EKF state dynamics

$$
\boldsymbol{X}(k)=\boldsymbol{X}(k-1)+\Delta T \boldsymbol{f}_{x}(\boldsymbol{X}(k-1), \boldsymbol{u}(k-1)),
$$

where $\Delta T=20 \mathrm{~ms}$ is the sampling period. The values of the rider-bicycle systems and the bias model parameters are listed in Table I. 
TABLE I

RIDER-BICYCLE MODEL AND NOISE MODEL PARAMETERS

\begin{tabular}{|c|c|c|c|c|c|c|c|c|c|c|c|c|c|}
\hline \hline$\alpha(\mathrm{deg})$ & $\gamma(\mathrm{deg})$ & $g\left(\mathrm{~m} / \mathrm{s}^{2}\right)$ & $l_{s}(\mathrm{~m})$ & $h_{s}(\mathrm{~m})$ & $l_{b}(\mathrm{~m})$ & $h_{b}(\mathrm{~m})$ & $p_{x}(\mathrm{~m})$ & $p_{z}(\mathrm{~m})$ & $L_{1}(\mathrm{~m})$ & $L_{x}(\mathrm{~m})$ & $L_{y}(\mathrm{~m})$ & $L_{m}(\mathrm{~m})$ & $\tau_{x}, \tau_{y}, \tau_{z}(\mathrm{~s})$ \\
\hline 10 & 20 & 9.8 & 0.26 & 0.38 & 0.45 & 0.66 & 0.37 & 0.71 & 0.13 & 0.2 & 0.12 & 0.043 & 1800 \\
\hline \hline
\end{tabular}

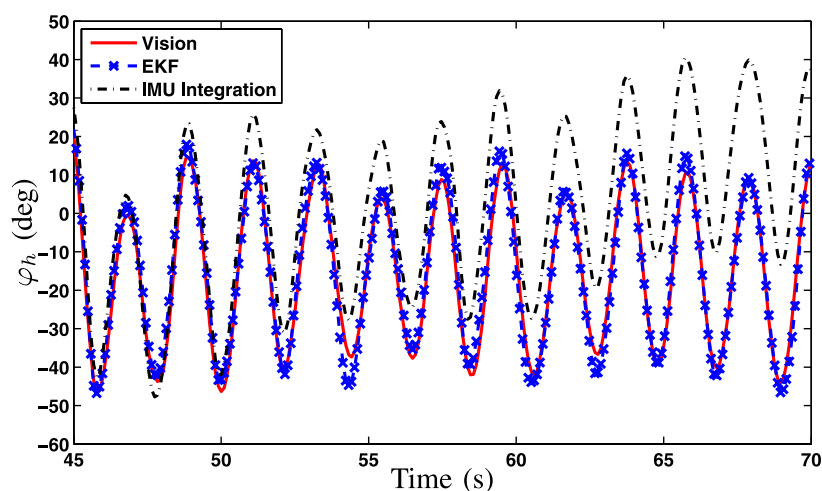

(a)

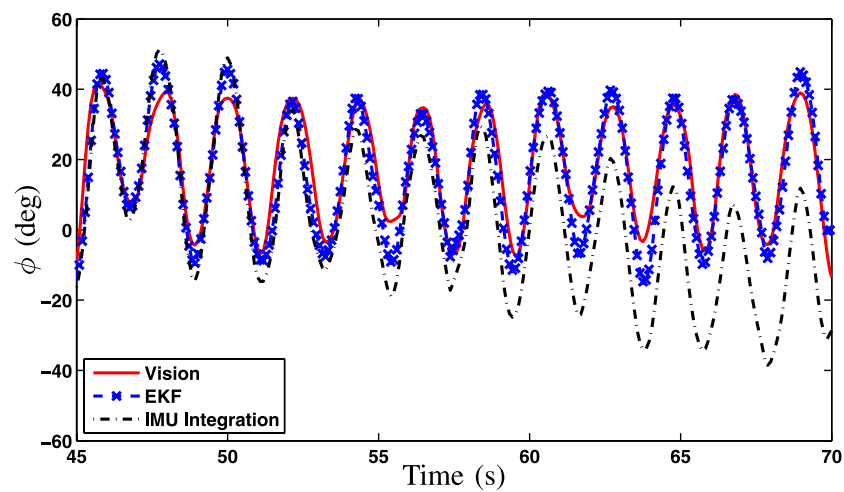

(c)

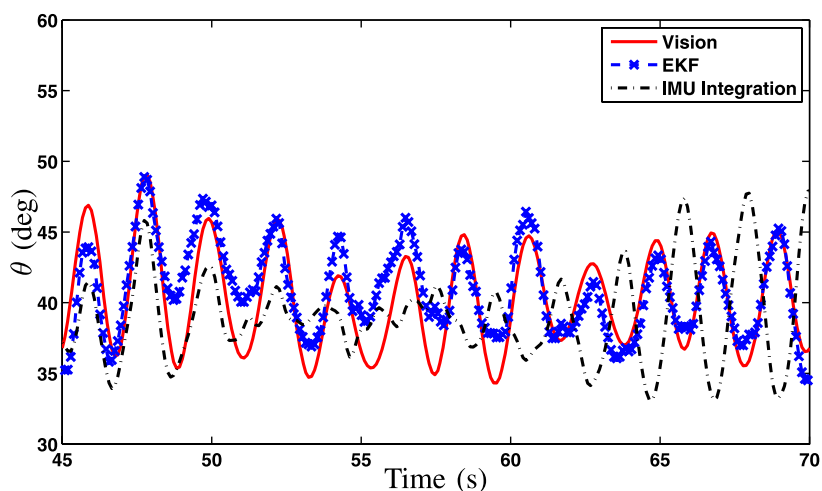

(b)

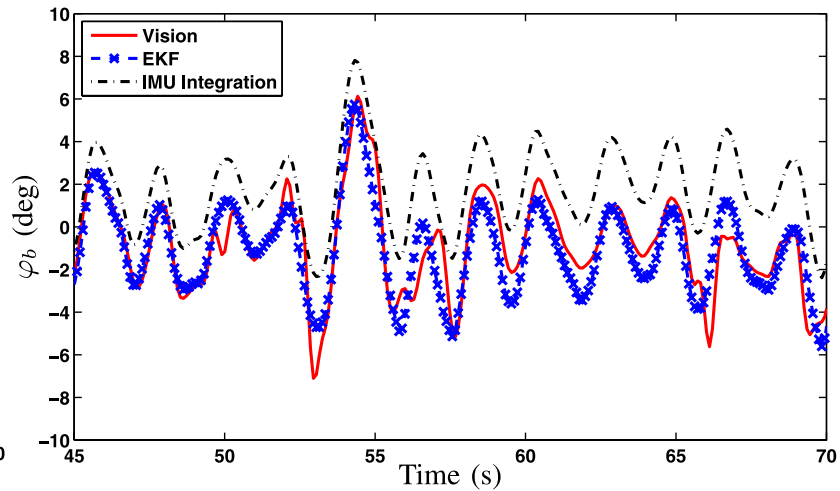

(d)

Fig. 6. Indoor comparison results of the estimated poses by the Vicon motion capturing systems, the EKF estimates, and the integrations of IMU measurements for Subject \#1. (a) $\varphi_{h}$. (b) $\theta$. (c) $\phi$. (d) $\varphi_{b}$.

For EKF outputs, we obtain the estimated torques $\hat{\tau}_{\varphi_{h}}$ and $\hat{\tau}_{\theta}$ in (11) and (12) and the estimated bicycle roll angle $\hat{\varphi}_{b}$ in (13). Moreover, the experiments show that the rider always tries to keep his/her shoulder level during bicycle riding. Therefore, a level-shoulder constraint among the trunk orientation angles is then used as a virtual measurement, namely,

$$
s_{c}=\mathrm{s}_{\varphi_{h}} \mathrm{c}_{\phi}+\mathrm{c}_{\varphi_{h}} \mathrm{c}_{\theta} \mathrm{s}_{\phi}=0
$$

Thus, including the above physical and virtual measurements, we have the EKF output equation

$$
\boldsymbol{y}(k)=\boldsymbol{h}(\boldsymbol{X}(k))+\boldsymbol{n}_{y}(k)=\left[\begin{array}{c}
\tau_{\varphi_{h}}(k) \\
\tau_{\theta}(k) \\
\varphi_{b}(k) \\
s_{c}(k)
\end{array}\right]+\boldsymbol{n}_{y}(k)
$$

The first two elements of $\boldsymbol{h}(\boldsymbol{X}(k))$ are calculated by (9) and $\boldsymbol{n}_{y}(k) \sim \mathcal{N}\left(\mathbf{0}, \boldsymbol{\Sigma}_{y}\right)$ is the white noise vector with variance matrix $\Sigma_{y}$. Given the system equation (16) and output equation
(18), the EKF design is similar to that in [32] and a brief description is given in Appendix B.

\section{RESULTS}

We recruited five healthy and experienced bicycle riders (four male and one female with age: $27 \pm 3$ years, height: $176 \pm 4 \mathrm{~cm}$, and weight: $70 \pm 7 \mathrm{~kg}$ ) to conduct both the indoor and the outdoor experiments. The duration for each riding experiment run was around $2 \mathrm{~min}$. When riding the bicycle, the subjects were asked to arbitrarily change the bicycle speed and trajectory and to freely move their upper bodies. In outdoor experiments, the maximum bicycle speed was around $22 \mathrm{~km} / \mathrm{h}$. All the subjects gave their informed consent before being tested using a protocol approved by the Institutional Review Board at Rutgers University.

Fig. 6 shows the indoor pose estimation results for Subject\#1. The subject rode the bicycle by his own riding style. Fig. 6(a)(c) shows the estimates of the trunk pose angles and Fig. 6(d) shows the estimates of the bicycle roll angle. For clarity, we 


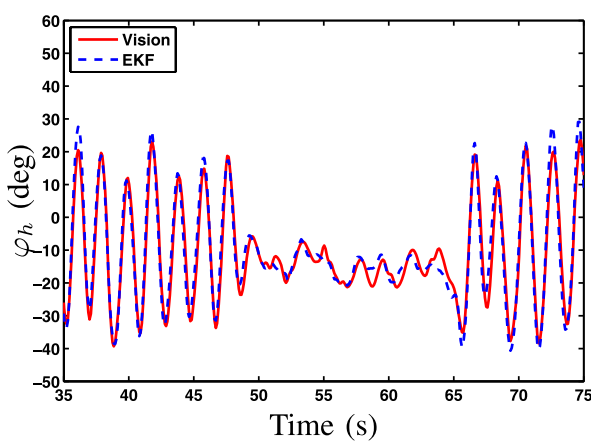

(a)

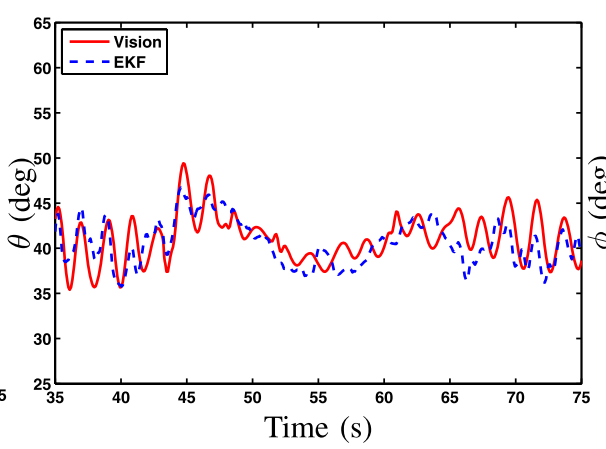

(b)

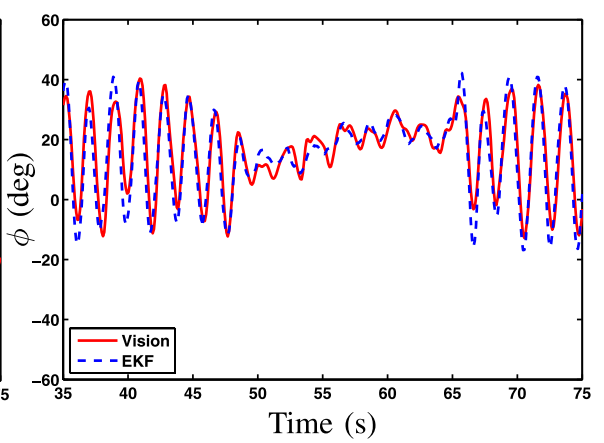

(c)

Fig. 7. Indoor comparison results of the estimated poses by the Vicon vision capturing systems, the EKF estimates for Subject \#2. (a) $\varphi_{h}$. (b) $\theta$. (c) $\phi$.

TABLE II

MEAN AND SD OF RMS ERRorS With AND Without THE BiAs MODEL (BM)

\begin{tabular}{|c|c|c|c|c|c|}
\hline \hline \multicolumn{2}{|c|}{ Experiment type } & $\varphi_{h}(\mathrm{deg})$ & $\theta(\mathrm{deg})$ & $\phi(\mathrm{deg})$ & $\varphi_{b}(\mathrm{deg})$ \\
\hline \multirow{2}{*}{ Indoor } & With BM & $3.42 \pm 1.34$ & $1.62 \pm 1.06$ & $5.56 \pm 1.45$ & $0.82 \pm 0.31$ \\
\cline { 2 - 6 } & No BM & $3.73 \pm 1.46$ & $1.84 \pm 1.09$ & $5.81 \pm 1.42$ & $0.84 \pm 0.33$ \\
\hline \multirow{2}{*}{ Outdoor } & With BM & $4.46 \pm 1.62$ & $4.01 \pm 1.61$ & $6.18 \pm 2.43$ & $2.31 \pm 0.89$ \\
\cline { 2 - 6 } & No BM & $4.42 \pm 1.75$ & $4.18 \pm 1.63$ & $6.24 \pm 2.39$ & $2.30 \pm 0.88$ \\
\hline \hline
\end{tabular}

only show truncated estimates during the time period between 45 to $70 \mathrm{~s}$. For comparison purposes, the estimates by the direct strapdown IMU integration are also plotted in these figures. For the direct IMU integration results, the offset biases (i.e., $c_{h}$ and $c_{b}$ in the noise model (14)) are measured and their effects are eliminated in calculation. The subject tried to move his trunk aggressively in the experiment. Balancing the bicycle for a counterclockwise circular trajectory, the subject tilted his trunk toward the center of the trajectory. Thus, the average value of $\varphi_{h}$ is around $-20^{\circ}$. The EKF-based estimation results clearly demonstrate a superior tracking performance than those by the direct strapdown IMU integration. The estimates by the direct IMU integration diverge after $50 \mathrm{~s}$ as shown in Fig. 6(a)(c), while the EKF-based pose estimates consistently match the ground truth. Fig. 7 shows the indoor estimation performance for Subject \#2. Comparing with Subject \#1, this subject moved his trunk arbitrarily. For example, as shown in Fig. 7, the subject moved his trunk aggressively before $48 \mathrm{~s}$, then mildly and slightly from 48 to $66 \mathrm{~s}$, and finally aggressively again. In this experiment, the EKF-based pose estimation also demonstrates matching performance. The pose estimation performance of the outdoor experiments demonstrates similar results as those of the indoor experiments.

To further demonstrate the performance of the EKF-based design, we compute the statistics of the pose estimation errors for all subjects. Table II shows the accuracy performance in terms of the mean and standard deviation (SD) of root mean square (RMS) errors for all subjects for both the indoor and the outdoor tests. Fig. 8 shows the calculated statistic errors over time for all subjects. For all experiments, the estimation errors are around zero and do not grow over time. The results shown in Table II and Fig. 8 confirm the consistently robust performance of the estimated trunk and bicycle poses by the EKF-based sensing fusion.

\section{DISCUSSIONS}

In [14], the fusion of inertial and magnetic sensors shows the mean RMS errors of $2.4^{\circ}$ to $3.2^{\circ}$ for different body segments orientations. The approach in [13] demonstrates a $2.8^{\circ}$ mean RMS error in the orientation estimation by using inclination in the Kalman filter design. In [19], the fusion of inertial and ultrasonic sensors demonstrates the mean RMS errors ranging from $5.7^{\circ}$ to $6.6^{\circ}$. All the above results are obtained in the laboratory conditions. Compared with those results, our indoor experiment results are on the same accuracy level. Although our outdoor experiments show slightly large errors, the accuracy is still comparable to those by the other fusion approaches.

From the results shown in Table II and Fig. 8, we notice that the estimation errors are not at the same level among the four estimated angles. In general, estimates of $\varphi_{b}$ have the least errors, while the estimates of $\phi$ have the largest errors. These differences are due to several reasons. First, during typical bicycle riding, the change of the bicycle roll angle $\varphi_{b}$ is relatively small. The performance of the IMU-based estimation within a small range is better than those with a large range such as for the trunk angles. Second, there is no direct measurement for angle $\phi$ and the constraint (17) of $\phi$ is coupled with the other two angles. Therefore, the estimation of $\phi$ has the maximal error. Moreover, for different riding styles, the performances of the EKF-based estimation design are not the same. For example, for a rider with an aggressively riding style, the EKF-based estimation scheme produces relatively larger errors than those with a mild riding style.

To quantify the improvement of the usage of the randomwalk bias model in the EKF design, we compare the estimation errors with and without the inclusion of the bias model. As shown in Table II, the results confirm that the use of the bias model improves the overall estimation performance. For outdoor experiments, the improvement is not significant due to the large ground truth errors provided by the onboard camera. We shall emphasize that, with or without using the bias model, the EKFbased fusion always achieves the nondrifting results, unlike the strapdown IMU integration approach. 


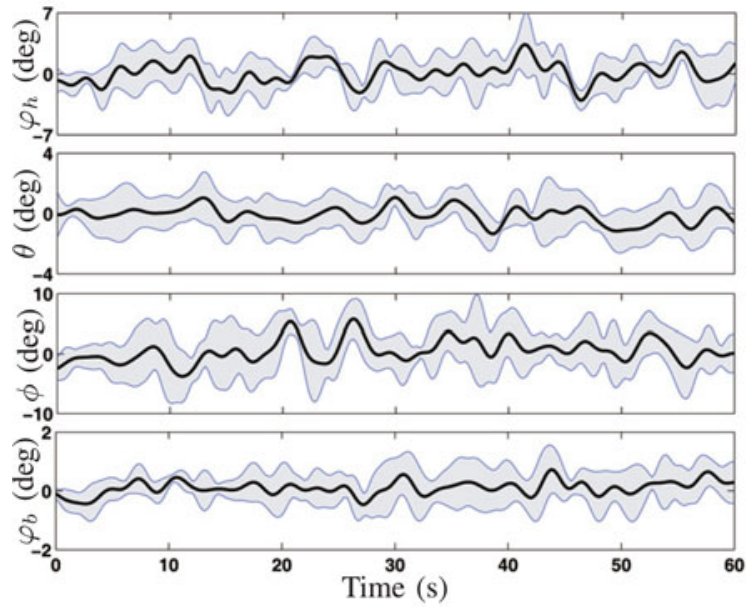

(a)

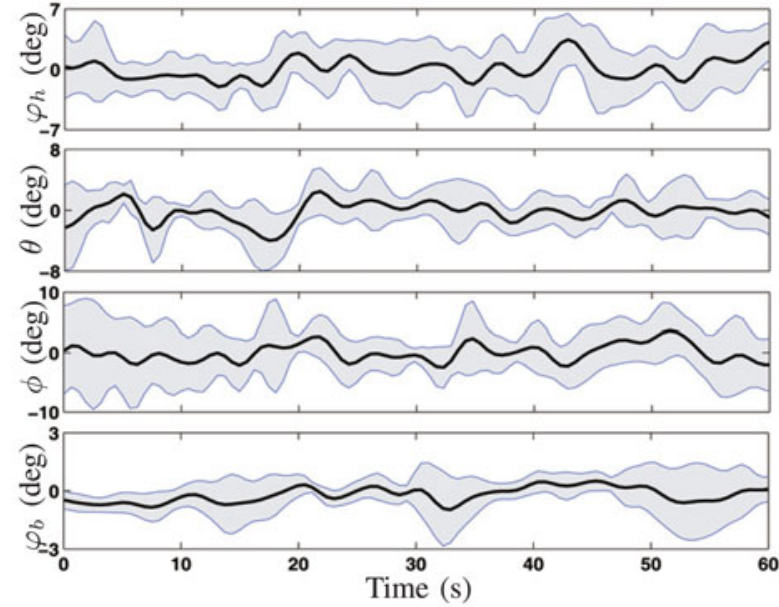

(b)

Fig. 8. Pose estimation errors from the EKF-based design. The solid lines indicate the mean values of the errors of all subjects and the dashed lines are one-SD bounds. (a) Indoor experiments. (b) Outdoor experiments.

The estimation errors are mainly due to the imperfect models for the IMU measurement noises, the interaction forces, and the complicated rider-bicycle interactions. For example, the calculated torques $\hat{\tau}_{\varphi_{h}}$ and $\hat{\tau}_{\theta}$ in (11) and (12) do not consider the articulated arm dynamics and the interactions between the legs and the trunk (e.g., pedaling effect). During the normal riding, we found the influences from the articulated arm dynamics and the pedaling effects are not significant for trunk pose estimation. Therefore, we ignore them in the rider-bicycle dynamic model for simplicity. Another error source might come from the measurement errors by the Vicon motion capture system. We estimated and found a maximum $0.8^{\circ}$ accuracy for the trunk pose angles and $0.3^{\circ}$ accuracy for the bicycle roll angle measurements by the Vicon motion capture system. For the outdoor experiments, the vibration of the camera mounting base due to uneven road surface also contributes to the ground truth errors.

Human trunk is flexible and its orientation is difficult to define and measure. In [26]-[28], the trunk is modeled as a rigid onelink inverted pendulum in walking, stance, and bicycling balance studies. To validate this rigid-body modeling assumption, we placed a set of optical markers along the spine to observe the characteristics of trunk motion (see Fig. 1(d)). Fig. 9 shows the means and the SDs of the spine curvature calculations in the sagittal plane and the coronal plane during one indoor riding experiment. The results shown in the figure confirm that the trunk maintains rigid-body motions in bicycle riding since the variations of the spinal curvatures are small. The curvatures at the level T4-T11 region, where the rider IMU is attached, are small. This implies a straight line-shape spine. Especially, in the coronal plane the curvature of the entire spine is around zero. Therefore, the rigid pendulum seems a valid model for capturing the trunk motion in bicycle riding.

The force measurements at the seat and handlebar contain motion information about the rider-bicycle pose angles and their dynamics, as shown in (9). The use of the IMU measurements facilitates the EKF design to calculate the pose information from the force measurements by (9). Therefore, the fusion of

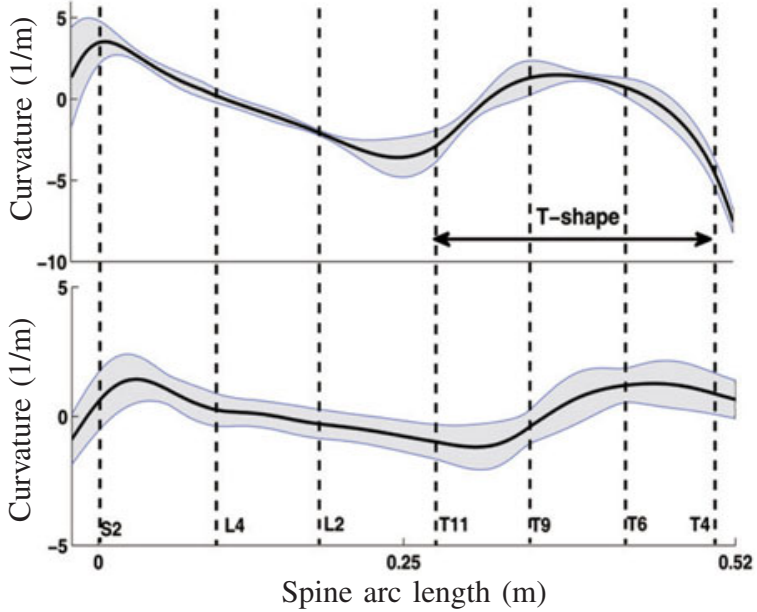

Fig. 9. Mean values and one-SD bounds of the spine curvatures in the sagittal plane (top) and in the coronal plane (bottom).

the force/inertial measurements in the EKF design provides a more restrictive constraint than that of the velocity constraints such as those in [31] and [32]. This is the main reason why the drifts by the strapdown IMU integration are eliminated. Unlike the approaches in [6] and [13] to use an empirical acceleration model accuracy enhancement, the use of force measurements is built on the system dynamics, and thus, the obtained estimation results are reliable under dynamic motions. The robust estimation results shown in Fig. 8 confirm such an observation.

Unlike some inertial sensor-based schemes in which the ground-truth pose information is needed to initialize the estimation design [20], the force/inertial fusion design is robust to the choice of the initial state values. For example, Fig. 10 shows the EKF-based estimate of $\varphi_{h}$ in the first few seconds. Although the initial value is set by more than $30^{\circ}$ different from its actual value, the estimates of $\varphi_{h}$ converge to the ground truth within 


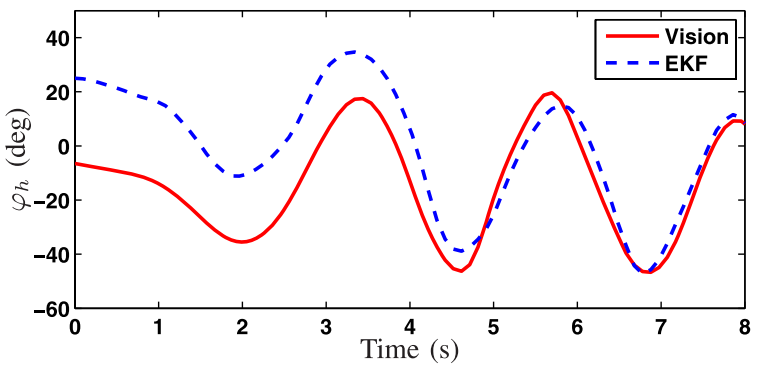

Fig. 10. Initialization and convergence of the EKF-based estimation design (only shown for $\varphi_{h}$ ).

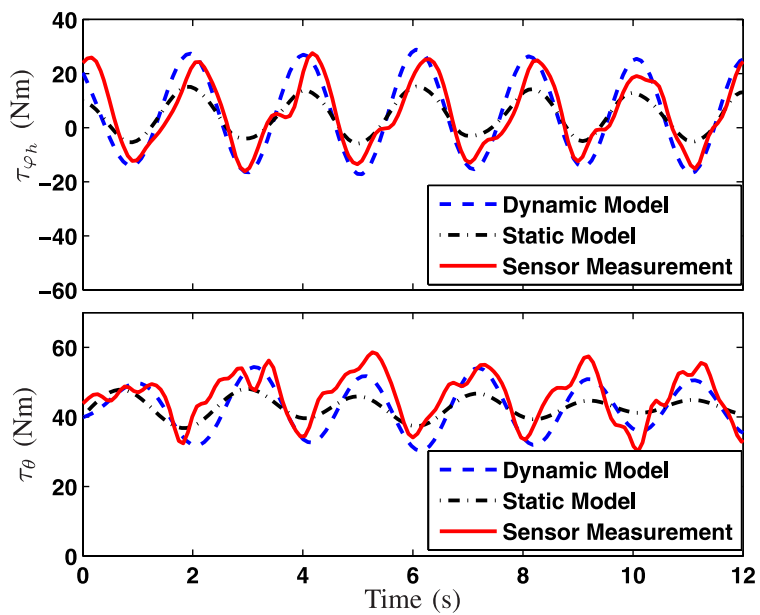

Fig. 11. Comparison results among the dynamic model-based, the static model-based, and the sensor measured $\tau_{\varphi_{h}}$ and $\tau_{\theta}$.

around $6 \mathrm{~s}$. Of course, it is preferable to use a set of initial values that are near their true values for a fast convergence. We used the static force measurements to calculate the initial values for the state variables [28]. The static model assumes zero derivatives in (9). Fig. 11 shows the comparison of the estimated torques by the dynamic model, the static model, and the sensor measurements. Clearly, the dynamic model-based torque calculations match well with the measurements, while the magnitudes of the static model-based calculations are underestimated because of the neglected dynamic effects. The results in Fig. 11 also indirectly validate the rider-bicycle dynamic model (9).

As one design limitation, the force/inertial sensor fusion design is not capable to identify and estimate the bicycle yaw angle $\psi$ because none of the onboard sensors provides the absolute yaw motion information. The used inverted pendulum model is valid only for typical bicycle riding in which the rider always sits on the seat. The pose estimation will not perform well in the cases of uncommonly riding styles, such as acrobatic riders who stand on the pedals and do not sit on the seat.

\section{CONCLUSION}

In this paper, we have presented a rider-bicycle pose estimation scheme using the inertial and force sensors. The pose estimation scheme was built on the attractive properties of the robust force measurements and the motion-sensitive responses of the inertial sensors. A rider-bicycle dynamic model was de- veloped to provide the underlying dynamic relationship between the pose angles and the human driving forces. By incorporating the rider-bicycle dynamics into the design, the pose estimation results did not drift over time even under highly dynamic motions. We demonstrated and validated the pose estimation scheme through both the indoor and the outdoor bicycle riding experiments. The results showed superior performance than those by using the direct strapdown IMU integration method and were also comparable with the other fusion methods in literature. Since the pose estimation approach uses only onboard sensors, it is particularly useful for outdoor applications and also potentially for other types of human-machine interactions.

\section{APPENDIX A}

\section{RIDER-BICYCLE DYNAMIC MODEL}

The rider-bicycle dynamic model is obtained by the Lagrangian approach. The Lagrangian of the rider-bicycle system is obtained as

$$
\begin{aligned}
\mathcal{L}= & \frac{1}{2} m_{b}^{\mathcal{R}} \boldsymbol{v}_{B} \cdot{ }^{\mathcal{R}} \boldsymbol{v}_{B}+\frac{1}{2} m_{h}^{\mathcal{R}} \boldsymbol{v}_{H} \cdot{ }^{\mathcal{R}} \boldsymbol{v}_{H} \\
& -g\left[m_{b} h_{b} \mathrm{c}_{\varphi_{b}}+m_{h}\left(h_{s} \mathrm{c}_{\varphi_{b}}+h_{h} \mathrm{c}_{\varphi_{h}} \mathrm{~s}_{\theta}\right)\right],
\end{aligned}
$$

where $m_{b}$ is the bicycle mass, and ${ }^{\mathcal{R}} \boldsymbol{v}_{B}$ and ${ }^{\mathcal{R}} \boldsymbol{v}_{H}$ are the linear velocities for the bicycle and the trunk, respectively. We obtain ${ }^{\mathcal{R}} \boldsymbol{v}_{B}=\left[\begin{array}{lll}v_{b} & 0 & 0\end{array}\right]^{T}+{ }^{\mathcal{R}} \boldsymbol{\omega}_{b} \times \boldsymbol{\rho}_{B}$. Similarly, we obtain the seat velocity ${ }^{\mathcal{R}} \boldsymbol{v}_{s}$ and ${ }^{\mathcal{R}} \boldsymbol{v}_{H}$ is then calculated as ${ }^{\mathcal{R}} \boldsymbol{v}_{H}={ }^{\mathcal{R}} \boldsymbol{v}_{s}+{ }^{\mathcal{R}}$ $\boldsymbol{\omega}_{h} \times \boldsymbol{\rho}_{H}$, where $\boldsymbol{\rho}_{H}=\left[\begin{array}{llll}h_{h} \mathrm{c}_{\theta} & h_{h} \mathrm{~s}_{\varphi_{h}} \mathrm{~s}_{\theta} & -h_{h} \mathrm{c}_{\varphi_{h}} \mathrm{~s}_{\theta}\end{array}\right]^{T}$. Finally, using Lagrange's equations, matrices $M$ and $G$ are obtained as

$$
\boldsymbol{M}=\left[\begin{array}{ccc}
m_{h} h_{h}^{2} \mathrm{~s}_{\theta}^{2} & 0 & 0 \\
0 & m_{h} h_{h}^{2} & 0
\end{array}\right], \boldsymbol{G}=\left[\begin{array}{c}
-m_{h} g h_{h} \mathrm{~s}_{\varphi_{h}} \mathrm{~s}_{\theta} \\
m_{h} g h_{h} \mathrm{c}_{\varphi_{h}} \mathrm{c}_{\theta}
\end{array}\right]
$$

and $\boldsymbol{C}\left(\boldsymbol{q}, \dot{\boldsymbol{q}}, \ddot{\boldsymbol{q}}_{b}\right)$ is given in (19) at the bottom of the next page.

\section{APPENDIX B}

\section{EKF IMPLEMENTATION}

An EKF design is applied to (16) and (18). For the state dynamics (16), we obtain the Jacobian matrix $\boldsymbol{F}(k)$ as

$$
\boldsymbol{F}(k)=\boldsymbol{I}_{7}+\Delta T \boldsymbol{F}_{X}(k),
$$

where $\boldsymbol{I}_{n}$ is $n \times n$ identity matrix and $\boldsymbol{F}_{X}(k)=\left.\frac{\partial \boldsymbol{f}_{x}}{\partial \boldsymbol{X}}\right|_{\boldsymbol{X}(k), \boldsymbol{u}(k)}$. The final result for $\boldsymbol{F}_{X}(k)$ is given in (20) at the bottom of the next page. Similarly, the Jacobian matrix $\boldsymbol{H}(k)=$ $\left.\frac{\partial \boldsymbol{h}}{\partial \boldsymbol{X}}\right|_{\boldsymbol{X}(k), \boldsymbol{u}(k)}$ is obtained for outputs (18) as

$$
\boldsymbol{H}(k)=\left[\begin{array}{ccccccc}
H_{11} & H_{12} & 0 & H_{14} & 0 & 0 & 0 \\
H_{21} & H_{22} & 0 & H_{24} & 0 & 0 & 0 \\
0 & 0 & 0 & 1 & 0 & 0 & 0 \\
H_{41} & H_{42} & H_{43} & 0 & 0 & 0 & 0
\end{array}\right],
$$


where elements of $\boldsymbol{H}(k)$ are given in (21) at the bottom of the page. Note that the IMU measurements are used to calculate $\boldsymbol{F}_{X}(k)$ and $\boldsymbol{H}(k)$ through (4), (3), (7), and (8).

With these Jacobian matrices, the EKF implementation is written as a prediction step $(\hat{\boldsymbol{X}}(k \mid k-1))$ and a correction step $(\hat{\boldsymbol{X}}(k \mid k))$ recursively. A similar design is given in [32] and we omit the details here.

\section{REFERENCES}

[1] S. R. Simon, "Quantification of human motion: Gait analysis-Benefits and limitations to its application to clinical problems," J. Biomech., vol. 37 , no. 12, pp. 1869-1880, 2004.

[2] P. Bonato, "Wearable sensors and systems from enabling technology to clinical applications," IEEE Eng. Med. Biol. Mag., vol. 29, no. 3, pp. 2536, May/Jun. 2010

[3] R. A. Hyde, L. P. Ketteringham, S. A. Neild, and R. J. S. Jones, "Estimation of upper-limb orientation based on accelerometer and gyroscope measurements," IEEE Trans. Biomed. Eng., vol. 55, no. 2, pp. 746-754, Feb. 2008.

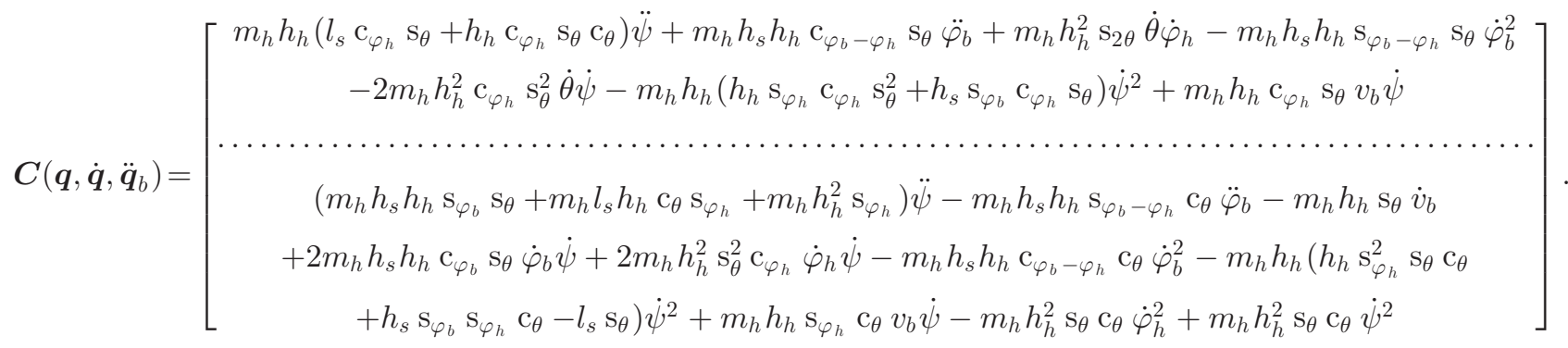

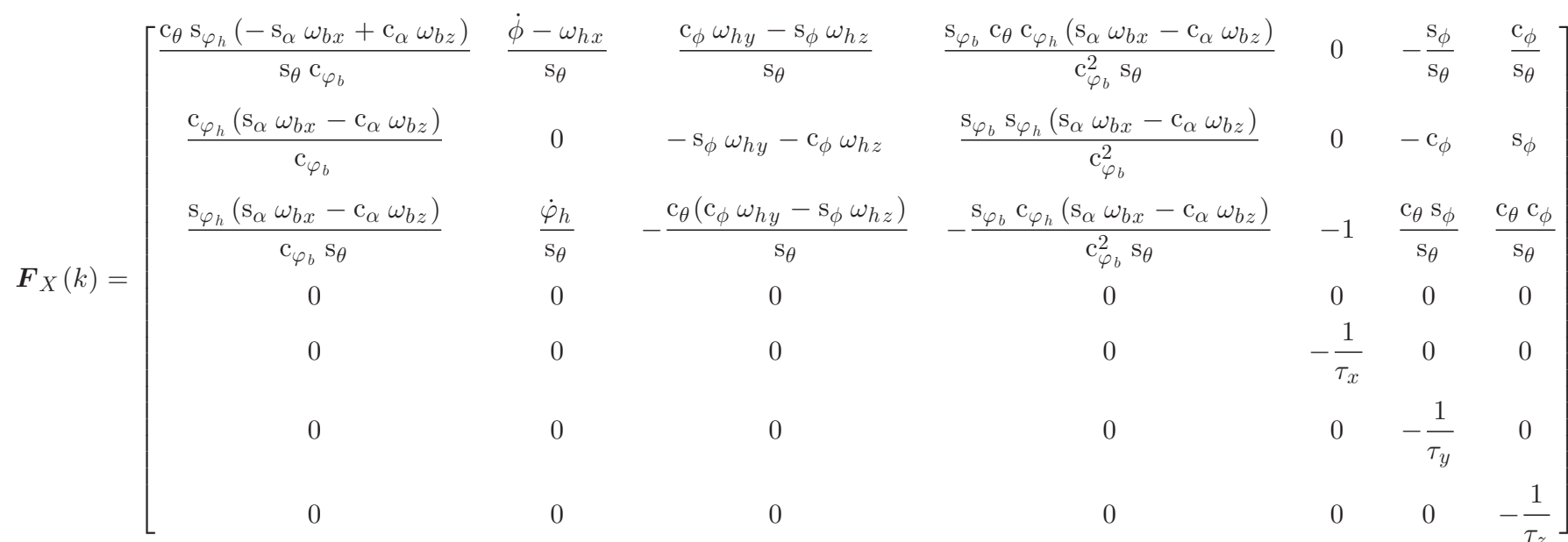

$$
\begin{aligned}
& H_{11}=-m_{h} h_{h}\left(l_{s} \mathrm{~s}_{\varphi_{h}} \mathrm{~s}_{\theta}+h_{h} \mathrm{~s}_{\varphi_{h}} \mathrm{~s}_{\theta} \mathrm{c}_{\theta}\right) \ddot{\psi}+m_{h} h_{s} h_{h} \mathrm{c}_{\varphi_{b}-\varphi_{h}} \mathrm{~s}_{\theta} \dot{\varphi}_{b}^{2}+2 m_{h} h_{h}^{2} \mathrm{~s}_{\varphi_{h}} \mathrm{~s}_{\theta}^{2} \dot{\theta} \dot{\psi} \\
& +m_{h} h_{h}\left(-h_{h} \mathrm{c}_{2 \varphi_{h}} \mathrm{~s}_{\theta}^{2}+h_{s} \mathrm{~s}_{\varphi_{b}} \mathrm{~s}_{\varphi_{h}} \mathrm{~s}_{\theta}\right) \dot{\psi}^{2}-m_{h} h_{h} \mathrm{~s}_{\varphi_{h}} \mathrm{~s}_{\theta} v_{b} \dot{\psi}-m_{h} g h_{h} \mathrm{c}_{\varphi_{h}} \mathrm{~s}_{\theta} \text {, } \\
& H_{12}=2 m_{h} h_{h}^{2} \mathrm{~s}_{\theta} \mathrm{c}_{\theta} \ddot{\varphi}_{h}+m_{h} h_{h}\left(l_{s} \mathrm{c}_{\varphi_{h}} \mathrm{c}_{\theta}+h_{h} \mathrm{c}_{\varphi_{h}} \mathrm{c}_{2 \theta}\right) \ddot{\psi}+2 m_{h} h_{h}^{2} \mathrm{c}_{2 \theta} \dot{\theta} \dot{\varphi}_{h}-m_{h} h_{s} h_{h} \mathrm{~s}_{\varphi_{b}-\varphi_{h}} \mathrm{c}_{\theta} \dot{\varphi}_{b}^{2} \\
& -2 m_{h} h_{h}^{2} \mathrm{c}_{\varphi_{h}} \mathrm{~s}_{2 \theta} \dot{\theta} \dot{\psi}-m_{h} h_{h}\left(h_{h} \mathrm{~s}_{\varphi_{h}} \mathrm{c}_{\varphi_{h}} \mathrm{~s}_{2 \theta}+h_{s} \mathrm{~s}_{\varphi_{b}} \mathrm{c}_{\varphi_{h}} \mathrm{c}_{\theta}\right) \dot{\psi}^{2}+m_{h} h_{h} \mathrm{c}_{\varphi_{h}} \mathrm{c}_{\theta} v_{b} \dot{\psi}-m_{h} g h_{h} \mathrm{~s}_{\varphi_{h}} \mathrm{c}_{\theta} \text {, } \\
& H_{21}=\left(m_{h} l_{s} h_{h} \mathrm{c}_{\theta} \mathrm{c}_{\varphi_{h}}+m_{h} h_{h}^{2} \mathrm{c}_{\varphi_{h}}\right) \ddot{\psi}-2 m_{h} h_{h}^{2} \mathrm{~s}_{\theta}^{2} \mathrm{~s}_{\varphi_{h}} \dot{\varphi}_{h} \dot{\psi}-m_{h} h_{s} h_{h} \mathrm{~s}_{\varphi_{b}-\varphi_{h}} \mathrm{c}_{\theta} \dot{\varphi}_{b}^{2} \\
& -m_{h} h_{h}\left(h_{h} \mathrm{~s}_{2 \varphi_{h}} \mathrm{~s}_{\theta} \mathrm{c}_{\theta}+h_{s} \mathrm{~s}_{\varphi_{b}} \mathrm{c}_{\varphi_{h}} \mathrm{c}_{\theta}\right) \dot{\psi}^{2}+m_{h} h_{h} \mathrm{c}_{\varphi_{h}} \mathrm{c}_{\theta} v_{b} \dot{\psi}-m_{h} g h_{h} \mathrm{~s}_{\varphi_{h}} \mathrm{c}_{\theta} \\
& H_{22}=\left(m_{h} h_{s} h_{h} \mathrm{~s}_{\varphi_{b}} \mathrm{c}_{\theta}-m_{h} l_{s} h_{h} \mathrm{~s}_{\theta} \mathrm{s}_{\varphi_{h}}\right) \ddot{\psi}-m_{h} h_{h} \mathrm{c}_{\theta} \dot{v}_{b}+2 m_{h} h_{s} h_{h} \mathrm{c}_{\varphi_{b}} \mathrm{c}_{\theta} \dot{\varphi}{ }_{b} \dot{\psi}+2 m_{h} h_{h}^{2} \mathrm{~s}_{2 \theta} \mathrm{c}_{\varphi_{h}} \dot{\varphi}_{h} \dot{\psi} \\
& +m_{h} h_{s} h_{h} \mathrm{c}_{\varphi_{b}-\varphi_{h}} \mathrm{~s}_{\theta} \dot{\varphi}_{b}^{2}-m_{h} h_{h}\left(h_{h} \mathrm{~s}_{\varphi_{h}}^{2} \mathrm{c}_{2 \theta}-h_{s} \mathrm{~s}_{\varphi_{b}} \mathrm{~s}_{\varphi_{h}} \mathrm{~s}_{\theta}-l_{s} \mathrm{c}_{\theta}\right) \dot{\psi}^{2}-m_{h} h_{h} \mathrm{~s}_{\varphi_{h}} \mathrm{~s}_{\theta} v_{b} \dot{\psi} \\
& -m_{h} h_{h}^{2} \mathrm{c}_{2 \theta} \dot{\varphi}_{h}^{2}+m_{h} h_{h}^{2} \mathrm{c}_{2 \theta} \dot{\psi}^{2}-m_{h} g h_{h} \mathrm{c}_{\varphi_{h}} \mathrm{~s}_{\theta}, \\
& H_{24}=m_{h} h_{s} h_{h} \mathrm{c}_{\varphi_{b}} \mathrm{~s}_{\theta} \ddot{\psi}-2 m_{h} h_{s} h_{h} \mathrm{~s}_{\varphi_{b}} \mathrm{~s}_{\theta} \dot{\varphi}_{b} \dot{\psi}+m_{h} h_{s} h_{h} \mathrm{~s}_{\varphi_{b}-\varphi_{h}} \mathrm{c}_{\theta} \dot{\varphi}_{b}^{2}-m_{h} h_{h} h_{s} \mathrm{c}_{\varphi_{b}} \mathrm{~s}_{\varphi_{h}} \mathrm{c}_{\theta} \dot{\psi}^{2} \\
& H_{14}=-2 m_{h} h_{s} h_{h} \mathrm{~s}_{\varphi_{b}-\varphi_{h}} \mathrm{~s}_{\theta} \dot{\varphi}_{b}, H_{41}=\mathrm{c}_{\varphi_{h}} \mathrm{c}_{\phi}-\mathrm{s}_{\varphi_{h}} \mathrm{c}_{\theta} \mathrm{s}_{\phi}, H_{42}=-\mathrm{s}_{\theta} \mathrm{c}_{\varphi_{h}} \mathrm{~s}_{\phi}, H_{43}=-\mathrm{s}_{\varphi_{h}} \mathrm{~s}_{\phi}+\mathrm{c}_{\varphi_{h}} \mathrm{c}_{\theta} \mathrm{c}_{\phi} \text {. }
\end{aligned}
$$


[4] Z.-Q. Zhang, W.-C. Wong, and J.-K. Wu, "Ubiquitous human upper-limb motion estimation using wearable sensors," IEEE Trans. Inf. Technol. Biomed., vol. 15, no. 4, pp. 513-521, Jul. 2011.

[5] H. J. Luinge, P. H. Veltink, and C. T. M. Baten, "Ambulatory measurement of arm orientation," J. Biomech., vol. 40, no. 1, pp. 78-85, 2007.

[6] H. Zhou and H. Hu, "Reducing drifts in the inertial measurements of wrist and elbow positions," IEEE Trans. Instrum. Meas., vol. 59, no. 3, pp. 575-585, Mar. 2010.

[7] H. Dejnabadi, B. M. Jolles, E. Casanova, P. Fua, and K. Aminian, "Estimation and visualization of sagittal kinematics of lower limbs orientation using body-fixed sensors," IEEE Trans. Biomed. Eng., vol. 53, no. 7, pp. 1385-1393, Jul. 2006.

[8] A. M. Sabatini, C. Martelloni, S. Scapellato, and F. Cavallo, "Assessment of walking features from foot inertial sensing," IEEE Trans. Biomed. Eng., vol. 52, no. 3, pp. 486-494, Mar. 2005.

[9] H. M. Schepers, H. F. J. M. Koopman, and P. H. Veltink, "Ambulatory assessment of ankle and foot dynamics," IEEE Trans. Biomed. Eng., vol. 54, no. 5, pp. 895-902, May 2007.

[10] K. J. O'Donovan, R. Kamnik, D. T. O'Keeffe, and G. M. Lyons, "An inertial and magnetic sensor based technique for joint angle measurement," J. Biomech., vol. 40, no. 12, pp. 2604-2611, 2007.

[11] K. Liu, Y. Inoue, K. Shibata, and E. Cao, "Ambulatory estimation of kneejoint kinematics in anatomical coordinate system using accelerometers and magnetometers," IEEE Trans. Biomed. Eng., vol. 58, no. 2, pp. 435-442, Feb. 2011.

[12] R. Zhu and Z. Zhou, "A real-time articulated human motion tracking using tri-axis inertial/magnetic sensors package," IEEE Trans. Neural Syst. Rehab. Eng., vol. 12, no. 2, pp. 295-302, Jun. 2004.

[13] H. J. Luinge and P. H. Veltink, "Measuring orientation of human body segments using miniature gyroscopes and accelerometers," Med. Biol. Eng. Comput., vol. 43, no. 2, pp. 273-282, 2005.

[14] D. Roetenberg, P. J. Slycke, and P. H. Veltink, "Ambulatory position and orientation tracking fusing magnetic and inertial sensing," IEEE Trans. Biomed. Eng., vol. 54, no. 5, pp. 883-890, May 2007.

[15] A. M. Sabatini, "Quaternion-based extended Kalman filter for determining orientation by inertial and magnetic sensing," IEEE Trans. Biomed. Eng., vol. 53, no. 7, pp. 1346-1356, Jul. 2006.

[16] C.-G. Song, J.-Y. Kim, and N.-G. Kim, "A new postural balance control system for rehabilitation training based on virtual cycling," IEEE Trans. Inf. Technol. Biomed., vol. 8, no. 2, pp. 200-207, Jun. 2004.

[17] M. B. Aerts, W. F. Abdo, and B. R. Bloem, "The "bicycle sign" for atypical Parkinsonism," Lancet, vol. 377, no. 9760, pp. 125-126, 2011.

[18] A. H. Snijders and B. R. Bloem, "Cycling for dreezing of gait," N. Engl. J. Med., vol. 362, p. e46, 2010.

[19] D. Vlasic, R. Adelsberger, G. Vannucci, J. Barnwell, M. Gross, W. Matusik, and J. Popović, "Practical motion capture in everyday surroundings," ACM Trans. Graph., vol. 26, no. 3, 2007, article 35.

[20] Q. Li, M. Young, V. Naing, and J. M. Donelan, "Walking speed estimation using a shank-mounted inertial measurement unit," J. Biomech., vol. 43, no. 8, pp. 1640-1643, 2010.

[21] H. Rouhani, J. Favre, X. Crevoisier, and K. Aminian, "Ambulatory measurement of ankle kinetics for clinical applications," J. Biomech., vol. 44, pp. 2712-2718, 2011

[22] P. H. Veltink, C. Liedtke, E. Droog, and H. van der Kooij, "Ambulatory measurement of ground reaction forces," IEEE Trans. Neural Syst. Rehab. Eng., vol. 13, no. 3, pp. 423-427, Sep. 2005.

[23] H. M. Schepers, E. H. F. van Asseldonk, J. H. Buurke, and P. H. Veltink, "Ambulatory estimation of center of mass displacement during walking," IEEE Trans. Biomed. Eng., vol. 56, no. 4, pp. 1189-1195, Apr. 2009.

[24] P. H. Veltink, H. Kortier, and H. M. Schepers, "Sensing power transfer between the human body and the environment," IEEE Trans. Biomed. Eng., vol. 56, no. 6, pp. 1711-1718, Jun. 2009.

[25] Y. Zhang, K. Chen, and J. Yi, "Dynamic rider/bicycle pose estimation with force/IMU measurements," presented at the Amer. Control Conf., Washington, DC, USA, 2013.

[26] A. D. Goodworth and R. J. Peterka, "Influence of bilateral vestibular loss on spinal stabilization in humans," J. Neurophysiol., vol. 103, no. 4, pp. 1978-1987, 2010

[27] J. H. van der Spek, P. H. Veltink, H. J. Hermens, B. F. J. M. Koopman, and H. B. K. Boom, "A model-based approach to stabilizing crutch supported paraplegic standing by artificial hip joint stiffness," IEEE Trans. Neural Syst. Rehab. Eng., vol. 11, no. 4, pp. 443-451, Jun. 2003.

[28] Y. Zhang, F. Liu, M. Trkov, and J. Yi, "Rider/bicycle pose estimation with IMU/seat force measurements," in Proc. IEEE/ASME Int. Conf. Adv. Intell. Mechatron., Kaohsiung, Taiwan, Jul. 2012, pp. 604-609.
[29] X. Lu, Y. Zhang, K. Yu, J. Yi, and J. Liu, "Upper limb pose estimation in rider-bicycle interactions with an un-calibrated monocular camera and wearable gyroscopes," presented at the ASME Dyn. Syst. Control Conf., Stanford, CA, 2013.

[30] R. Murray, Z. Li, and S. Sastry, A Mathematical Introduction to Robotic Manipulation. Boca Raton, FL, USA: CRC Press, 1994.

[31] C. C. Ward and K. Iagnemma, "A dynamic-model-based wheel slip detector for mobile robots on outdoor terrain," IEEE Trans. Robot., vol. 24 no. 4, pp. 821-831, Aug. 2008.

[32] J. Yi, H. Wang, J. Zhang, D. Song, S. Jayasuriya, and J. Liu, "Kinematic modeling and analysis of skid-steered mobile robots with applications to low-cost inertial-measurement-unit-based motion estimation," IEEE Trans. Robot., vol. 25, no. 5, pp. 1087-1097, Oct. 2009.

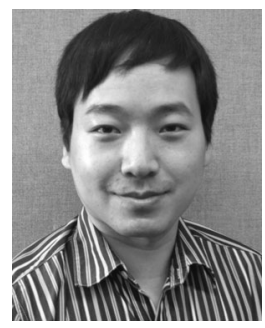

Yizhai Zhang (S'11) received the B.S. degree and the M.S. degree in information and communication engineering from Xi' an Jiaotong University, China, in 2005 and 2009, respectively. He is currently working toward the $\mathrm{Ph} . \mathrm{D}$. degree in mechanical and aerospace engineering at Rutgers University, Piscataway, NJ, USA.

His current research interests include autonomous robotic systems, dynamic systems and control, intelligent sensing and actuation systems, and mechatronics.

Mr. Zhang is a student member of ASME. He received the Best Student Paper at the 2012 IEEE/ASME International Conference on Advanced Intelligen Mechatronics and the 2012 ASME Dynamic Systems and Control Conference.

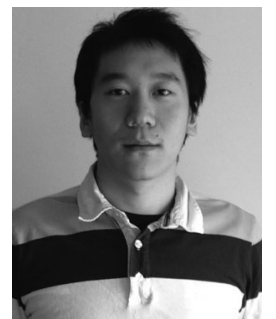

Kuo Chen received the B.S. degree in information technical science from Nankai University, China, in 2011. He is currently working toward the Ph.D. degree in mechanical and aerospace engineering at Rutgers University, Piscataway, NJ, USA.

His research interests include physical humanrobot interactions (HRI), dynamic systems and control, and machine learning with applications to robotics and HRI.

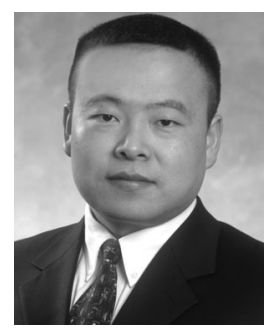

Jingang Yi (S'99-M'02-SM'07) received the B.S. degree in electrical engineering from Zhejiang University, Hangzhou, China, in 1993, the M.Eng. degree in precision instruments from Tsinghua University, Beijing, China, in 1996, the M.A. degree in mathematics and the Ph.D. degree in mechanical engineering from the University of California, Berkeley, CA, USA, in 2001 and 2002, respectively.

He is currently an Associate Professor of Mechanical Engineering at Rutgers University. His research interests include autonomous robotic systems, dynamic systems and control, mechatronics, automation science and engineering, with applications to biomedical systems, civil infrastructural, and transportation systems.

Dr. Yi is a member of American Society of Mechanical Engineers (ASME). $\mathrm{He}$ is a recipient of the 2010 US NSF CAREER Award. He has co-authored papers that have been awarded several best papers at the IEEE/ASME AIM, ASME DSCC, IEEE ICRA etc. He currently serves as an Associate Editor for the IEEE TRANSACTIONS ON AUTOMATION SCIENCE AND ENGINEERING and the IEEE Robotics and Automation Society Conference Editorial Board (since 2008). He also served as a Guest Editor for the IEEE TRANSACTIONS ON AUTOMATION SCIENCE AND ENGINEERING in 2009 and an Associate Editor for the ASME Dynamic Systems and Control Division Conference Editorial Board from 2008 to 2010. 